\title{
Field Strength Measurements in Fresh Water ${ }^{1}$
}

\author{
Gurdip S. Saran ${ }^{2}$ and Gedalia Held ${ }^{3}$
}

(February 26, 1960; revised April 5, 1960)

\begin{abstract}
Experiments were performed to measure field strength at a frequency of 18.6 kilocycles per second in fresh water of conductivity $2.66 \times 10^{-3}$ mhos/meter down to depths of 1,000 feet using monopole and loop antennas. The experimental results verify the theoretical values of field strength attenuation with depth for all antennas and of the ratio of vertical to horizontal field strength for the monopole antennas.
\end{abstract}

\section{Introduction}

The theoretical relationships of field strength in air and in the conducting medium for different antennas imbedded in the semi-infinite conducting medium have been presented by different authors [1 to 7]. ${ }^{4}$ M. B. Kraichman [8] has presented experimental results of the measurements of magnetic field strength in air for the various dipoles and loops immersed in a concentrated sodium chloride solution, which are in agreement with the above-referenced theoretical work. His experimental results, however, are limited in the horizontal range to a distance of over a wavelength in the conducting medium but a fraction of wavelength in air. Experiments are now in progress at Boeing Airplane Company to verify the theoretical results of electric field strength in air and in the conducting medium for the horizontal electric dipole in salt water for distances of several wavelengths in air.

Unfortunately the model-scale experiments performed in a laboratory are generally limited in scope due to the small dimensions of the tank, lead lengths, and the scaling factors that are introduced to fit the experiments to the limited space. Therefore full-scale experiments were performed. They are described briefly in this paper.

The experiments were performed at Lake Chelan in the State of Washington during the summer of 1959. The attenuation of field strength for different antennas down to depths of 1,000 feet was measured, and the field strength relationship between the horizontal and vertical monopoles was established experimentally. The conductivity of the water was $2.66 \times 10^{-3} \mathrm{mhos} / \mathrm{meter}$, which is comparable to that of the ground. The large size and great depth of the lake simplify considerably the experiments with regard to configurations and orientations of the antennas.

${ }^{1}$ Contribution from Boeing Airplane Company, Seattle, Wash., paper presented at Conference on the Propagation of ELF Radio Waves, Boulder, Colo.,

January 25, 1960
2 Present address, Space Sciences Laboratory, Missile and Space Vehicle Dept., General Electric Co., 3750 D St., Philadelphia, Pa.

${ }_{3}^{3}$ Present address, Space Technology Laboratories, Inc., P. O. Box 95001, Los Angeles 45, Calif.

4 Figures in brackets indicate the literature references at the end of this paper.
The signals were received from the U.S. Navy Radio Station (T) Jim Creek, Oso, Washington, at a frequency of $18.6 \mathrm{kc} / \mathrm{s}$. The field strength at different places on the surface of the lake was nearly 40 millivolts per meter.

The antennas tested were a 10 -ft loop with 15 turns, a 6 -ft loop with 15 turns, and a $36-\mathrm{ft}$ short-circuited coaxial antenna with $88-\mathrm{ft}$ bare wire for grounding to the water. The short-circuited coaxial antenna consists of an insulated section and a bare-wire section for grounding to the water [2]. All antennas were made of wire gage No. 4 with neoprene insulation.

\section{Theoretical Results}

The theoretical results of field strength for the electric and magnetic dipole antennas imbedded in the conducting medium are of the following general form:

$$
\psi=C P g(f, \sigma) \exp .\{-z / \delta\} h(f, r, \phi) .
$$

$C=$ a constant,

$P=$ electric or magnetic dipole moment,

$\sigma=$ conductivity in mhos/meter,

$z=\operatorname{sum}$ of depths for the transmitting and the receiving antennas,

$\delta=\left[2 / \omega \mu_{0} \sigma\right]^{1 / 2}$, skin depth,

$f=$ frequency,

$r, \phi=$ range and azimuth.

While the theoretical results involve different assumptions in the solution, they all suggest that the field strength attenuates exponentially with depth for the different antennas in the conducting medium. The exponential variation, furthermore, is invariant to the mode of excitation and reception for the antennas, as long as the distances involved are much greater than a skin depth [5]. The behavior of appropriate electromagnetic waves, therefore, is similar to that of a plane wave propagating in the conducting medium. For attenuation measurements in the lake water, therefore, only the relative amplitude of the electromagnetic wave was measured and is of importance. 
The ratio of vertical to horizontal component of the electric field strength in the conducting medium for a plane wave propagating along the interface is:

$$
\eta=E_{\mathrm{v}} / E_{n}=\left[\omega \epsilon_{0} / i \sigma\right]^{1 / 2}
$$

For a wave propagating in water, the vertical component is negligible compared to the horizontal component of electric field strength. The index of refraction of water is determined by the conductivity and is large compared to that of air. The direction of propagation of a wave in the water, therefore, is nearly vertical [9].

\section{Experimental Apparatus and Procedure, and Results}

\subsection{Apparatus and Procedure}

The apparatus consisted of a wooden raft, a small boat, a tank, antennas, and the receiving equipment consisting of batteries, converter, recorder, and a timer. The metal tank was used to accommodate the receiving equipment and could withstand pressure down to water depth of $1,500 \mathrm{ft}$. The test antennas were kept at a distance of nearly $12 \mathrm{ft}$ from the tank. The tank and antennas were supported by a nylon rope from a wooden raft. A barge was used only to handle the weight of the tank when the latter was out of the water. During the experiments, however, the barge was taken away and kept at a distance of at least $2,000 \mathrm{ft}$ from the test site. The timer inside the tank was set to start the receiving equipment approximately $12 \mathrm{~min}$ before the end of each hour and to shut off the equipment a few minutes past the hour. During the equipment-on period, the signals from the Naval Radio Station followed this sequence: a period of a few minutes with no signal followed by a period of approximately 5 min with cw power. During the cw signals, the antenna depth was varied with the rope on the wooden raft. The signals were also monitored in the air with another receiver on the raft to notice possible variation in field strength and to check the period of $\mathrm{cw}$ power from the radio station.

\subsection{Experimental Results}

The attenuation of field strength with depth for the monopole and the loop antennas are presented in figure 1. The field strength follows an exponential law as $E=E_{0}$ exp. $\{-z / \delta\}$. At one skin depth (72 meters for this case), for example, the field strength decreases to $1 / e$ of its value at the interface of air and water. The field strength, furthermore, decreases to $1 / 10$ and $1 / 100$ of its interface value at the depths of 164 and 329 meters respectively.

The vertical component is smaller than the horizontal component of the field strength. The experimental ratio of vertical to horizontal components is $0.0141,0.0138$, and 0.0149 for the respective depths of 3,10 , and 50 meters.

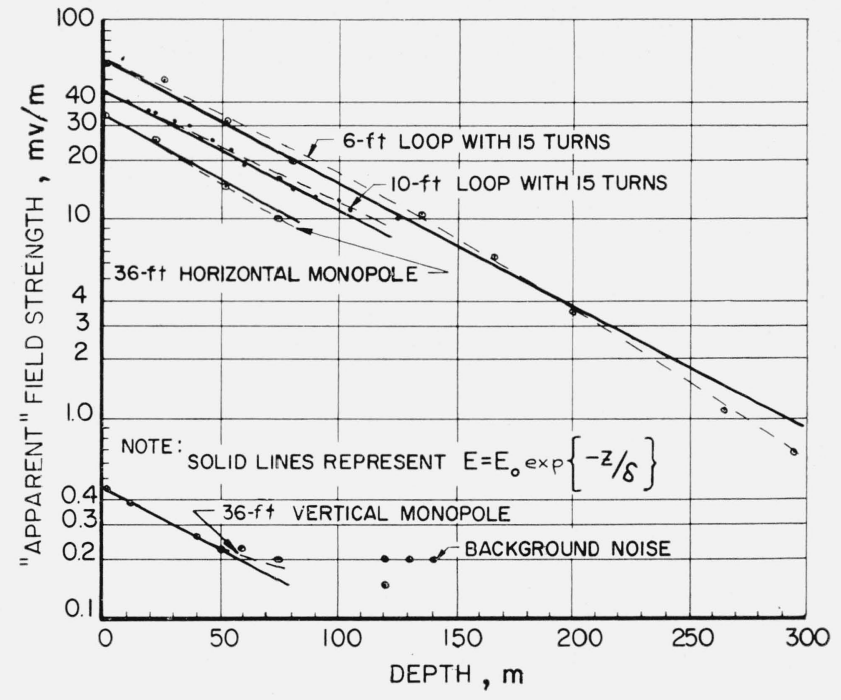

Figure 1. Attenuation of field strength with depth for the electric and magnetic dipole antennas.

\section{Discussion of Results}

The experimental values of field strength with depth correlated closely with those of theoretical values represented by $E=E_{0} \exp \{-z / \delta\}$. Precautions were taken to keep metal objects, including the barge, at least a few thousand feet away from the test site and the wooden raft. In order to a void any questions about the transmission of the signal over lines or wire, only nylon rope (no wires) was used to lower, raise, or handle the receiving equipment in the water.

The theoretical ratio of vertical to horizontal components of the field strength in the conducting medium is 0.0197 for the frequency of $18.6 \mathrm{kc} / \mathrm{s}$ and conductivity of $2.66 \times 10^{-3} \mathrm{mhos} / \mathrm{meter}$. The experimental values are lower than the theoretical results and are presented in table 1.

TABLE 1.- $\eta$, Ratio of vertical to horizontal component of $\mathrm{E}$ in water

\begin{tabular}{c|c|c}
\hline \hline $\begin{array}{c}\text { Depth in } \\
\text { meters }\end{array}$ & $\begin{array}{c}\text { Experimental } \\
\eta\end{array}$ & $\begin{array}{c}\text { Theoretical } \\
\eta\end{array}$ \\
\cline { 2 - 3 } & & 0.0141 \\
3 & .0138 & 0.0197 \\
10 & .0149 & .0197 \\
50 & .0197 \\
\hline
\end{tabular}

The discrepancy of 20 to 30 percent could have been caused by the change of azimuth angle and test site during experiments. The strong winds, particularly during these tests, on the lake could easily have moved the raft at least a mile away from the original test site. The change in both the azimuth angle and test site can influence results; the former because of transmitter location and the latter because of the variation of field strength with location at the surface of the lake. 


\section{Conclusions}

The experimental results for the attenuation of field strength with depth for the different antennas correlate with the theoretical results. The experiments, furthermore, verified the theoretical results that the horizontal electric antennas are superior to the vertical antennas for reception, and also for radiation (by reciprocity theorem), in the conducting medium.

\section{References}

[1] C. T. Tai, Hertzian dipole immersed in a dissipative medium, Cruft Lab. Rept. No. 21, Harvard Univ. (1947).

[2] R. K. Moore, The theory of radio communication between submerged submarines, Ph. D. Thesis, Cornell Univ. (1951).

[3] W. VonAulock, Low frequency electromagnetic dipole fields in a semi-infinite conductor, Navy Dept., Bureau of Ships, Minesweeping Sec., Tech. Rept., No. 104 (1952)
[4] J. R. Wait, Magnetic dipole antenna in a conducting medium, Proc. IRE 40, 1244 (1952).

[5] J. R. Wait and L. L. Campbell, The fields of an oscillating magnetic dipole immersed in a semi-infinite conducting medium, J. Geophys. Research 58, 167 (1953).

[6] R. H. Lien and J. R. Wait, Radiation from a horizontal dipole in a semi-infinite dissipative medium, J. Appl. Phys. 24, 1 to 5, 958 to 959 (1953).

[7] A. Baños, Jr., and J. P. Wesley, The horizontal electric dipole in a conducting half-space, Pt. I, Univ. Calif., Marine Phys. Lab., Scripps Inst. Oceanography, SIO Ref. 53-33 (Sept. 1953); Pt. II, SIO Ref. 54-31 (Aug. 1954).

[8] M. B. Kraichman, Basic experimental studies of the magnetic field from electromagnetic sources immersed in a semi-infinite conducting medium, J. Research NBS 64D, 21 (1960).

[9] H. A. Wheeler, Fundamental limitations of a small VLF antenna for submarines. IRE Trans. on Antennas and Propagation, Ap-6, 123 (1958).

(Paper 64D5-78) 